\title{
The Study of the FIB Induced Damage in Diamond
}

\author{
S. Rubanov ${ }^{*}$ and A. Suvorova ${ }^{* *}$ \\ *Electron Microscope Unit, Bio21 Institute, University of Melbourne, Victoria 3010, Australia \\ ${ }^{* *}$ Centre for Microscopy, Characterisation and Analysis, University of WA, Crawley 6009, Australia
}

Despite diamond's extreme properties, a cross-sectional TEM sample of diamond can be prepared using FIB technique in a few hours [1]. However, FIB milling has an unavoidable result in formation of the damage layers [2-3]. It was also shown that the FIB milling in silicon may result in formation of the polycrystalline phase inside the damage due to the local heating of the milling area [3]. Similar heating of the damage layer in diamond may result in its partial or full graphitisation. Apparently, the presence of the graphitic phase in the damage can significantly complicate TEM analysis of the diamond samples. So, the knowledge of the damage induced in diamond during FIB milling is critical for the TEM imaging. In this work the damage after FIB milling of the synthetic diamond at different ion energies were studied using conventional and analytical TEM.

FEI Nova 200 dual beam FIB system was used for damage formation in diamond and TEM sample preparation [4]. To study the initial stages of the damage formation diamond sample was irradiated with $30 \mathrm{keV} \mathrm{Ga}$ ions with fluence of $8 \times 10^{15}$ ions $/ \mathrm{cm}^{2}$ and then carbon coated to reduce charging. The damage area is clearly visible in Fig. 1a between crystalline diamond and carbon film. EELS examination showed the presence of both $\mathrm{sp}^{2}$ and $\mathrm{sp}^{3}$ bonding in the damage corresponding to two different phases of carbon. However, the absence of any diffraction contrast indicates that this damage layer is amorphous in structure. The surface step height above the damage area and damage layer thickness were measured to be $13 \mathrm{~nm}$ and $36 \mathrm{~nm}$. It is apparent that swelling occurred during amorphisation of diamond. Using a mass balance calculation the density of the amorphous damage layer was determined to be $2.25 \mathrm{~g} / \mathrm{cm}^{3}$ (20\% sp $\mathrm{s}^{3}$ fraction). There is also visible a dark diffraction contour in diamond near the amorphous-crystalline interface in Fig. 1a (indicated by arrow). The concentration of point defects here is below the amorphisation threshold but high enough to cause local crystal lattice distortion. Fig. 1b shows g-3g weak beam dark field image of the same area. The distorted area is visible as a bright white band with thickness $\sim 8 \mathrm{~nm}$. This "crystalline" damage can aggravate the sharpness of atomic columns in high resolution images.

Also, rectangular trenches $4 \times 4 \mu^{2}$ and $2 \mu \mathrm{m}$ deep were milled in diamond sample using $\sim 100 \mathrm{pA}$ $\mathrm{Ga}^{+}$ion beam current at 5, 10 and $30 \mathrm{keV}$ energies. After FIB milling the near surface regions of the trenches contain two types of damage: the bottom-wall damage where the ion beam was normal to the surface and the side-wall damage where it was at low angle to the trench walls. Fig. 2 (a-c) show cross-sectional images of the side-wall damage layers created during 5, 10 and $30 \mathrm{keV}$ FIB milling. The thicknesses of the amorphous damage layers were measured to be 3.5, 5 and $16 \mathrm{~nm}$ for sidewalls and 10.5, 15 and $44 \mathrm{~nm}$ for the bottom-walls. The TEM sample preparation process was optimised by using $5 \mathrm{keV}$ FIB cleaning milling on the final stage. HREM images of the (110) diamond lattice after $5 \mathrm{keV}$ cleaning are shown in Fig. 3. The amorphous damage layer reduction after $5 \mathrm{keV}$ cleaning results in the lower background noise in the HREM image. The "crystalline damage" area is also narrower which results in much sharper image of the atomic columns. Fig. 3b,c show the higher magnification and digitally filtered images of the diamond sample after $5 \mathrm{keV}$ FIB milling. The individual atomic columns (dumbbells) with spacing $0.089 \mathrm{~nm}$ are clearly visible. 
Thus, the use of $5 \mathrm{keV}$ FIB cleaning for the TEM diamond samples drastically improved the quality of the lattice images and allowed to resolve individual atomic column with sub-angstrom spacing.

\section{References}

[1] D.P. Hickey et al., J.Vac.Sci.Technol. A 24 (2006) 1302.

[2] J.F. Walker and R.F. Broom, Inst.Phys.Conf.Ser. 157 (1997) 473.

[3] S. Rubanov and. P.R. Munroe, J. Microsc. 214 (2004) 213.

[4] L.A. Giannuzzi et al., Microsc. Res. Tech. 41 (1998) 285.

[5] This work was supported by the Australian Research Council, under the Linkage International program. The authors gratefully acknowledge the use of the Titan microscope at the Electron Microscopy Centre at Technion University, Haifa, Israel and the help of Dr. Yaron Kauffmann.

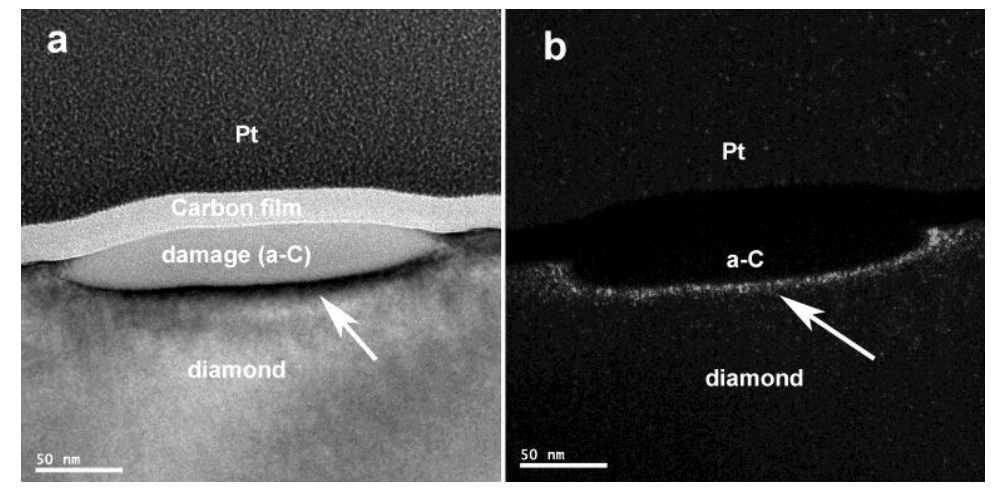

FIG. 1. Cross-sectional bright field (a) and weak beam dark field (b) images of the damage area in diamond after $30 \mathrm{keV}$ Ga FIB irradiation with fluence $8 \times 10^{15}$ ions $/ \mathrm{cm}^{2} ; \mathbf{g}=[2-20]$.
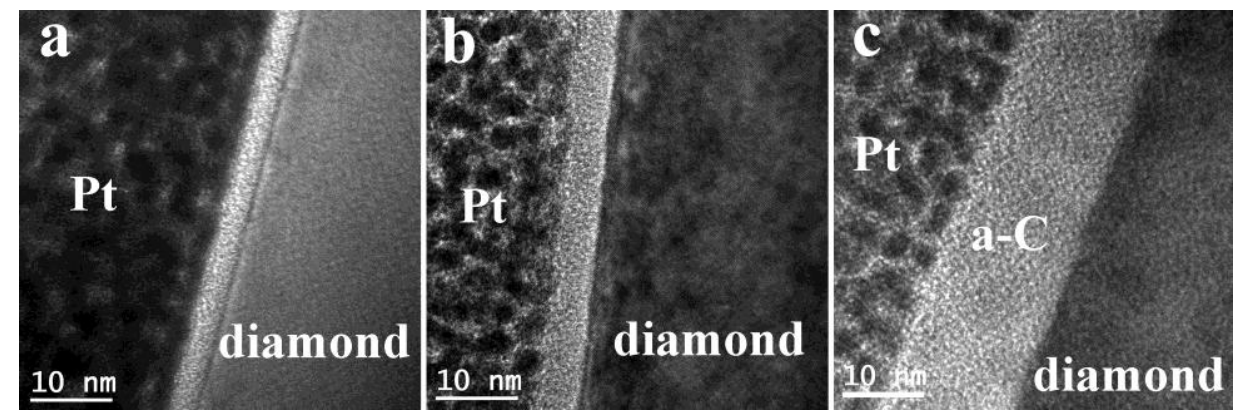

FIG. 2. Cross-sectional images of the side-wall damage layers in the diamond samples after FIB milling with 5 (a), 10 (b) and 30 (c) $\mathrm{keV} \mathrm{Ga}^{+}$ions.
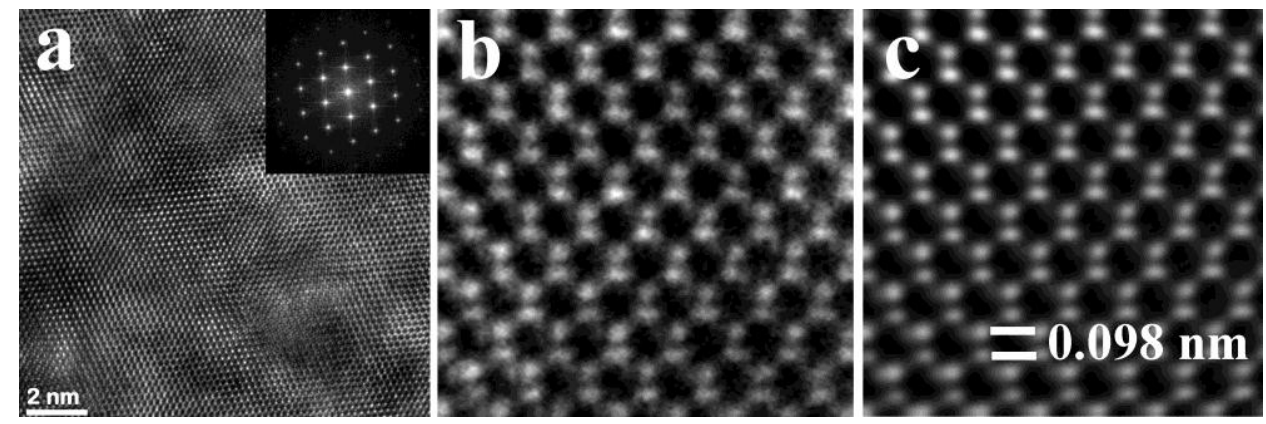

FIG. 3. HREM images of the (110) diamond lattice after $5 \mathrm{keV}$ FIB cleaning (a); magnified (b) and digitally filtered (b) images of the diamond lattice. 